\title{
Preparation of Polyacrylonitrile- based Porous Hollow Carbon Microspheres
}

\author{
Wei Han ${ }^{a}$, Shixiang Dong ${ }^{\mathrm{a}}, \mathrm{Bo} \mathrm{Li}^{\mathrm{b}^{*}}$, Liqin $\mathrm{Ge}^{\mathrm{a}^{*}}$ \\ ${ }^{a}$ State Key Laboratory of Bioelectronics, School of Biological Science and Medical \\ Engineering, Southeast University, Nanjing 210096, P. R. China \\ ${ }^{\mathrm{b}}$ Laser Fusion Research Center, China Academy of Engineering Physics, Mianyang \\ 621900, PR China
}

\begin{abstract}
:
Hollow carbon microspheres were successfully fabricated in a convenient fashion by employing polyacrylonitrile (PAN) emulsions as precursors. The obtained hollow carbon microspheres were shown high monodispersity, tunable pore structures -gradient pore diameters and uniform orientation of pores. Through varying the flowing rates of three phases and solidification time, the PAN microspheres with different sizes and densities could be simply synthesized. Besides the relationship between the solidification time and the density of microspheres as well as their gas barrier properties were studied in this paper. These structured microspheres have great potential applications in various areas, including foam targets, carbon molecular sieve, cargo storage, medical instruments and so on.
\end{abstract}

Keywords: Polyacrylonitrile; Hollow carbon microspheres; Microfluidic devices; Porous structure; Gas barrier properties

\footnotetext{
${ }^{*}$ Corresponding author. Tel.: +86 2583619983; fax: +86 2583795635.

E-mail address: lqge@ seu.edu.cn(L.Ge), lb6711@163.com(B. Li).
} 


\section{Introduction}

Recently, the preparation and application of hollow carbon microspheres $\left(\mathrm{HCM}_{\mathrm{S}}\right)$ have been a research hotspot of materials, especially $\mathrm{HCM}_{\mathrm{S}}$ with pore structures. $\mathrm{HCM}_{\mathrm{S}}$ have many fascinating properties, including high specific surface area, large pore volume, low density, good mechanical strength, electrical conductivity and high chemical inertness [1]. These innovative materials therefore present great utilitarian value for catalytic supports, adsorbents, energy storage, drug delivery, gas storage, nanoreactors and active material encapsulation and so on.

Researchers usually fabricate $\mathrm{HCM}_{\mathrm{S}}$ through chemical vapor deposition, template approach and hydrothermal method [2]. However, the template method has been proved to be the most effective method because of its simple operation, mild reaction condition, controllable thickness and size and so on. The template approach can be divided into hard template method and soft template method [2-5]. Hard template method has the disadvantage of poor mechanical strength, easy to damage structure after drying and removing templates, thus it is not suitable for the preparation of small microspheres [6]. Therefore soft template method is chosen to prepare $\mathrm{HCM}_{\mathrm{S}}$ by us. In addition, preparing porous materials is also a hotspot through shirasu porous glass-suspension polymerization, seeded swelling approach, traditional suspension polymerization and so on [7]. Porous polymer microspheres have been universally applied in dozens of fields such as the treatment on effluent, chemical industry, drug separation and purification, catalyst carrier and high performance liquid chromatography(HPLC) packing. Lots of researches designed to obtain porous 
materials were carried out with the help of different porogens [3,7-9]. To name a few, nitrogen-doped hollow carbon spheres with engineered large tunable mesoporous shells were successfully synthesized by using the diblock copolymer PS173-b-PEO170 [3], poly (ether sulfone) ultrafiltration membrane was got by using the amphiphilic additive Pluronic F127 [8]. Besides, other previous researches suggested that porous carbon materials could be obtained by using ultrasonic spray pyrolysis [10], redox reaction [11], high-temperature catalytic CVD [12], the breath figure technique [13] and other methods [14].

However, previous works without using porogens that combined $\mathrm{HCM}_{\mathrm{S}}$ with porous structures were rarely studied in recent years. Furthermore, the preparation of porous $\mathrm{HCM}_{\mathrm{S}}$ with narrow size distribution, regular shape and good monodispersity through a simple, easy and low-cost way is extremely tough. In addition, polyacrylonitrile (PAN) was considered as a promising low-cost carbon precursor in this contribution with the advantages of higher mechanical strength, lower deuterium-tritium permeation coefficient and remarkable anti-radiation $[15,16]$. To the best of our knowledge, the preparation of porous $\mathrm{HCM}_{\mathrm{S}}$ by microfluidics with PAN as the carbon source has not been thoroughly reported so far.

In this work, we present a highly reproducible fabrication method to prepare porous hollow microcapsules and carbon microspheres, which doesn't need porogens. PANbased porous hollow microcapsules and carbon microspheres with excellent monodispersity have been successfully obtained through a series of operations including solidification, rinsing with water, supercritical drying, pre-oxidation and 
carbonization (Fig. 1). We characterize and explore the microstructures and properties of PAN microspheres and carbon microspheres with different solidification time. Additionally, we simulated gas retention of PAN materials with the aim of hoping that PAN-based carbon microspheres could be used as foam targets. We find that PANbased porous hollow carbon microspheres have great gas barrier properties and the density of microspheres increases with solidification time.

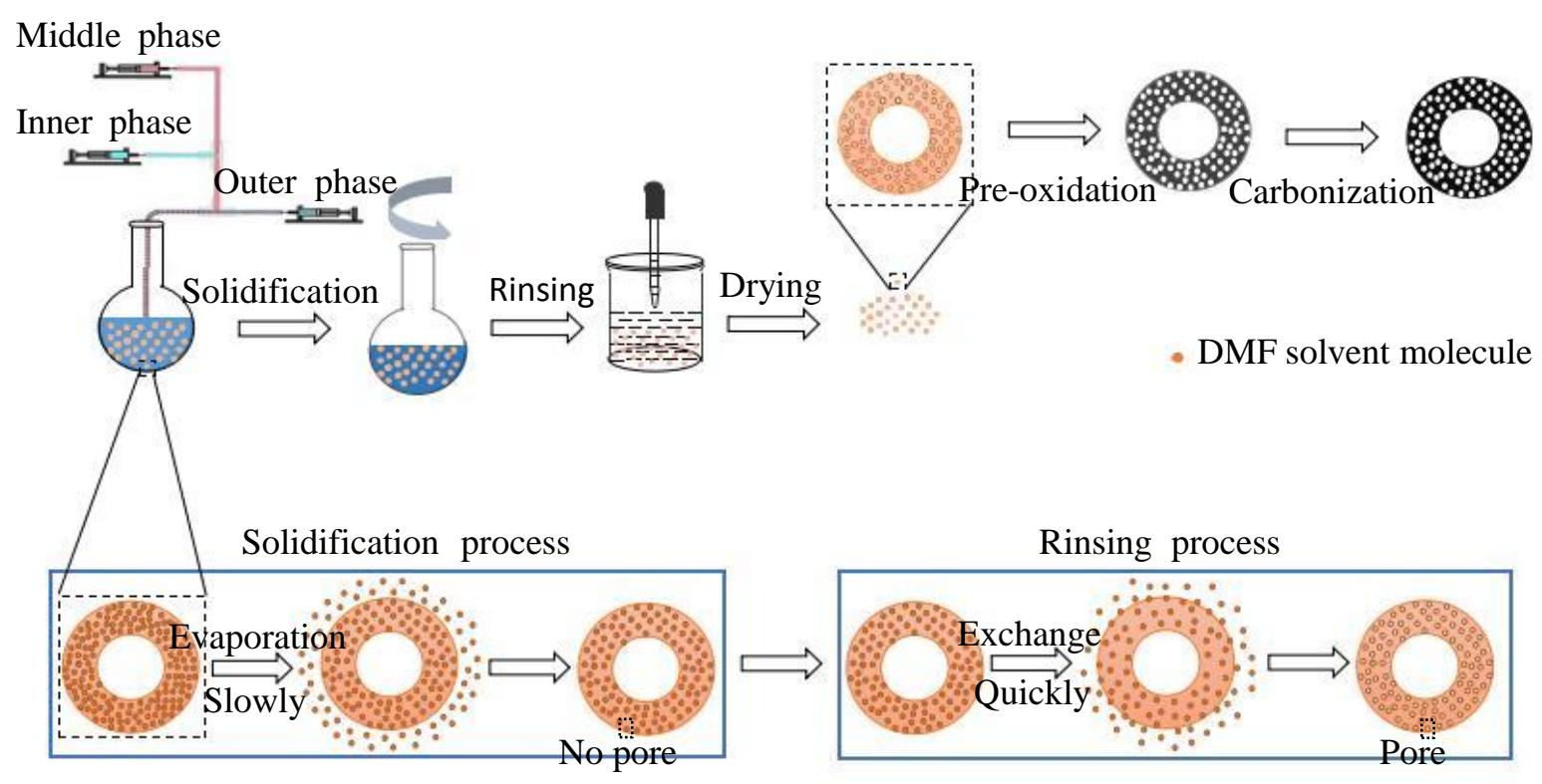

Fig.1. Scheme of preparation of PAN-based porous hollow carbon microspheres. Pictures in boxes are enlarged drawings.

\section{Experimental}

\subsection{Materials}

Polyacrylonitrile $(\mathrm{PAN}, \mathrm{Mw}=30,000)$ was purchased from Tri-high Membrane Technology Co. Ltd, China. Hexane, acetone, ethanol and N, N-dimethylformamide (DMF) were obtained from Sinopharm Chem-ical Reagent Shanghai Co. Ltd, P.R. 
China. The silicon oils $(\mathrm{KF}-96$, kinematic viscosity $=350 \mathrm{cSt}, 50 \mathrm{cSt}, 10 \mathrm{cSt}, 5 \mathrm{cSt}, 1 \mathrm{cSt}$, $0.65 \mathrm{cSt}$ ) were purchased from Shinetsu Chemical Co. Ltd., Japan. Deionized water was laboratory homemade. All the materials were used without further purification.

\subsection{Fabrication of hollow microcapsules}

In this process, a simple double T-junction microfludic device assembled in our group was used to obtain the PAN double emulsions, which were precursors of porous hollow carbon microspheres. The device had the advantage of simplicity, operability, controllability and cost-efficiency $[15,16]$. To generate monodisperse $\mathrm{O} / \mathrm{W}_{\mathrm{PAN} / \mathrm{O}}$ double emulsions, we used $50 \mathrm{cSt}$ silicon oil (KF-96) as the inner phase, $0.1 \mathrm{~g} / \mathrm{ml}$ PAN solution as the middle phase, and $50 \mathrm{cSt}$ silicon oil (KF-96) as the outer phase. These three flows were introduced and controlled by using three syringe pumps. Generated double emulsions were collected in $350 \mathrm{cSt}$ silicon oil (KF-96). To test the stability of double emulsions, the prepared double emulsions were collected and observed with optical stereo-scopic microscope.

After collecting generated double-emulsion droplets with a flask loaded with silicone oil, the flask was rested for 5 to 10 minutes and rotated in the speed range 35-45 rpm by rotary evaporator (RV10DS25, IKA) at room temperature. Several hours later, the microcapsules were rinsed by hexane, acetone, deionized water for several times, respectively, which removed DMF rapidly and generated pores in microcapsules. Then the samples were dried by heating through an oven or supercritical drying through critical point dryer (HCP-2, Hitachi) to remove inner silicon oil. Afterwards, the samples were pre-oxidated using muffle furnace (EP, 
ISUZU) through heating up to $300{ }^{\circ} \mathrm{C}$ with a rate of $2.5{ }^{\circ} \mathrm{C} / \mathrm{min}$ and then maintained for $2 \mathrm{~h}$ before cooled to room temperature in air atmosphere. Finally, the PAN microspheres were placed into the center of a tube furnace (Lindberg/Blue $\mathrm{M}$, Asheville) and carbonized through heating up to $800{ }^{\circ} \mathrm{C}$ with a rate of $10{ }^{\circ} \mathrm{C} / \mathrm{min}$ and maintained for $1 \mathrm{~h}$ before cooled to room temperature in an inert atmosphere.

\subsection{Characterization of microspheres}

Photographs of the microspheres were taken with optical stereo-scopic microscope (OLYMPUS U-LH75XEAPO, Tokyo, Japan) based on different amplification factors. The morphology of pores on samples were characterized by field emission scanning electron microscopy(SEM) operating at $0.8 \mathrm{kV}$ (Zeiss, Oberkochen, Germany).And the Raman spectroscopy measurement was carried out using QE65000 laser Raman spectrometer (OceanOptics, USA).

\subsection{Density of PAN microspheres}

The weight of dried hollow PAN microspheres was measured by electronic analysis balance (MS, METTLER TOLEDO). The outer radius and inner radius of hollow PAN microspheres rinsed by deionized water were measured by optical stereo-scopic microscope. The density was calculated by the following equations:

$$
\begin{aligned}
& \text { density }=\frac{\text { mass }}{\text { volume }}=\frac{\bar{m}}{\overline{\mathrm{V}}} \\
& \mathrm{V}=\frac{4 * \pi *\left(\mathrm{R}_{\mathrm{o}}{ }^{3}-\mathrm{R}_{\mathrm{i}}{ }^{3}\right)}{3}
\end{aligned}
$$

Where $R_{o}, R_{i}$ are the outer radius and inner radius of the PAN hollow microspheres, respectively. $\overline{\mathrm{m}}, \overline{\mathrm{V}}$ are the average mass and average volume of the PAN hollow microspheres, respectively. $\pi$ is approximately equal to 3.14 to calculate easily. 


\subsection{The permeability of PAN membranes}

The ceramic plate was immersed in $0.05 \mathrm{~g} / \mathrm{ml}$ PAN solution for $10-30 \mathrm{~min}$, and then put into an oven at $60{ }^{\circ} \mathrm{C}$ for $10-30$ min to dry. After generating PAN membranes, membrane of one side ceramic plate was sanded with sandpaper, while the other side ceramic plate was put into the Gas Permeation Device (Supplementary Fig. S1), where the radius of O-ring used was $15.50 \mathrm{~mm}$. Finally we measured the time taken for different kinds of gases to permeate to the membrane with the same volume of $1 \mathrm{ml}$ under the pressure of $0.1 \mathrm{M} \mathrm{Pa}$ at room temperature. The thickness of the membrane was got by calculating the average thickness of the membrane's different cross sections using SEM. The permeability coefficient is calculated by the following equations:

$$
\begin{aligned}
& \mathrm{P}_{\mathrm{V}}=\frac{\mathrm{m} * \mathrm{~d}}{\mathrm{~A} * \mathrm{t} * \mathrm{p}} \\
& \mathrm{n}=\frac{\mathrm{V}}{\mathrm{V}_{\mathrm{m}}}=\frac{\mathrm{m}}{\mathrm{M}}
\end{aligned}
$$

Where $P_{v}, m, d, A, t, p$ are the permeability coefficient, the mass of gas permeated, the thickness of the membrane, the area of gas pass through the membrane, the time of gas permeated, pressure difference on both sides of the membrane, respectively. $n$, $\mathrm{V}, \mathrm{M}, \mathrm{V}_{\mathrm{m}}$ are the amount of substance, the volume of gas permeated, molar volume of gas, molar mass, molar volume of gas, respectively.

\section{Results and discussion}

\subsection{Fabrication of PAN microcapsules.}

In order to avoid the influence of other molecules on pore structures such as the 
surfactant [15, 17, 18-20], surfactant-free PAN solution was prepared. Besides, during the process of using T-junction microfludic device to obtain the PAN double emulsions, it was important to obey the rule of density matching. As previous studies indicated that density matching was critical for the stabilization and concentricity of the double emulsions $[16,18,20]$, the density of $0.1 \mathrm{~g} / \mathrm{ml}$ PAN solution with $0.960 \mathrm{~g} / \mathrm{cm}^{3}$ was approximately equal to the density of $50 \mathrm{cSt}$ silicon oil (KF96) with $0.954 \mathrm{~g} / \mathrm{cm}^{3}$ (Supplementary, Table S1). So $50 \mathrm{cSt}$ KF96, 0.1 g/ml PAN solution and 50 cSt KF96 were used as the inner, middle and outer phases, respectively. In addition, the selection of collection phase was also of great importance. Previous reports had shown that with the increasing viscosity of collection phase, the emulsions became better monodispersed because of the balance between the shear force and the interfacial tension [21]. Therefore $350 \mathrm{cSt}$ KF96 was used as the collection phase to improve the monodispersity of products. A series of oil-in-water-in-oil (O/W/O) double emulsions ranging in size from micrometers to millimeters and thickness from tens of microns to hundreds of microns could be obtained by adjusting the flowing rates of three phase liquids [22-28]. In order to measure the weight and volume of microspheres easily, we preferred big emulsions with more than $500 \mu \mathrm{m}$ diameters (Fig. 2a). 

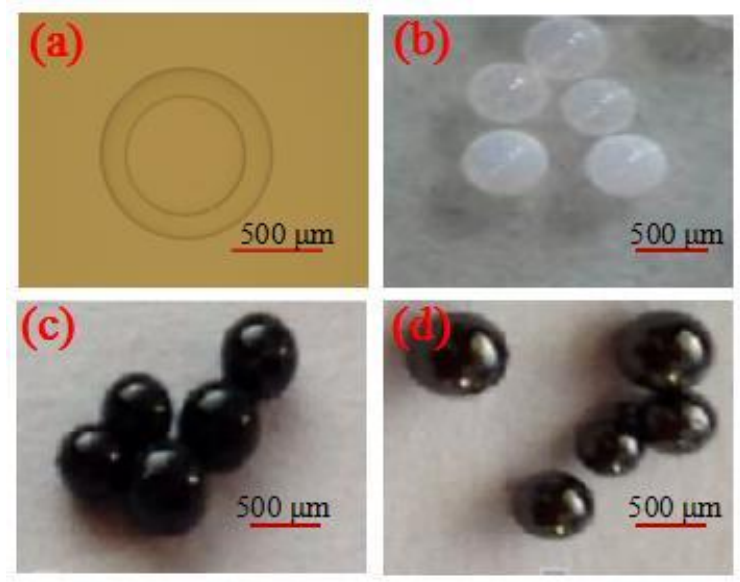

Fig.2. Photographs of PAN- based microspheres in different processes. (a) PAN double emulsions.

(b) Dried PAN microcapsules. (c) Pre-oxidated PAN microspheres. (d) PAN-based hollow carbon microspheres. Scale bars: $500 \mu \mathrm{m}$.

During the process of solidification, the resting time and rotary speed had important influences on the concentricity and sphericity of microcapsules [16]. After solidification for a period of time and water treatment, we could easily obtain the dried PAN hollow capsules, pre-oxidated PAN spheres and final carbon spheres (Fig. 2b-2d). It was shown that their colors were different -- the dried PAN hollow capsules were white or transparent, the pre-oxidized PAN hollow spheres were black, and the hollow carbon spheres were black and had a metallic luster.

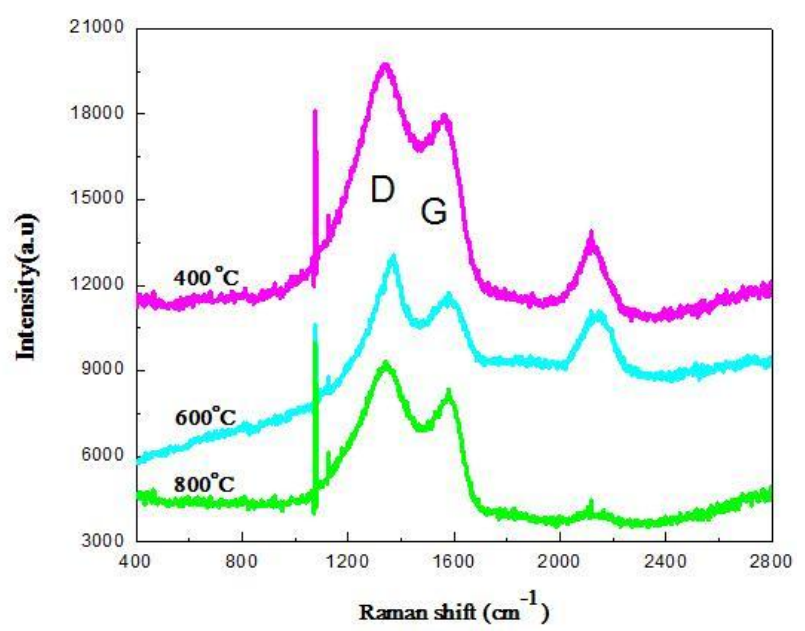

Fig.3. Raman spectrum of PAN- based hollow carbon microspheres under different 
carbonization temperatures.

The carbon states in hollow carbon spheres were identified by Raman spectroscopy. As shown in Fig. 3, the Raman spectra of PAN- based hollow carbon microspheres under different carbonization temperature showed two typical bands located at around $1350 \mathrm{~cm}^{-1}$ and $1580 \mathrm{~cm}^{-1}$, which corresponded to well-documented graphitic band (G) and the disordered band (D), respectively. The D band was generally related to the vibrations of $\mathrm{sp}^{3}$ hybridized carbon and defects in the plane terminations. And the $\mathrm{G}$ band was associated with vibrations of $\mathrm{sp}^{2}$-bonded ordered graphitic carbon sheets [3]. Thus, graphitic and disordered carbons co-existed in PAN-based hollow carbon microspheres. Besides, the ratio of $\mathrm{D}$ and $\mathrm{G}$ bands $\left(\mathrm{I}_{\mathrm{D}} / \mathrm{I}_{\mathrm{G}}\right)$ could be taken as a measurement of the relative crystallinity of a carbon material. The smaller ratio represented higher degree of graphitization and more complete structure. As shown in Table 1, the graphitization degree of the carbon spheres increased with carbonization temperature. So $800{ }^{\circ} \mathrm{C}$ was chosen as a proper carbonization temperature in the following experiments.

Table 1 Spectral data by Raman measurements for PAN- based hollow carbon microspheres under different carbonization temperature.

\begin{tabular}{|c|c|c|c|}
\hline Temperature & Center position of D peak $/ \mathrm{cm}^{-1}$ & Center position of $\mathrm{G}$ peak $/ \mathrm{cm}^{-1}$ & $\mathrm{I}_{\mathrm{D}} / \mathrm{I}_{\mathrm{G}}$ \\
\hline $400{ }^{\circ} \mathrm{C}$ & 1370 & 1580 & 1.15 \\
\hline $600{ }^{\circ} \mathrm{C}$ & 1341 & 1582 & 1.10 \\
\hline $800{ }^{\circ} \mathrm{C}$ & 1341 & 1581 & 1.07 \\
\hline
\end{tabular}




\subsection{The influence of different solidification time on PAN capsules and carbon}

spheres.

During the whole process of preparation, making DMF solvent quickly removed through shortening solidification time and then directly rinsing with water was the key to form porous structure. As shown in Fig. 1, in the process of rotating, the solvent of the double emulsions, DMF was evaporated. In DMF evaporation process, DMF was removed in a slow way, which could not leave pores in the solid shells. However, when directly rinsing with water, residual DMF in microcapsules was exchanged with water and removed quickly because DMF could be well soluble in water at any proportion, and therefore left a lot of pores.

At the beginning we selected a 24-hour period to explore the relationship between solidification time and density of PAN hollow capsules, because $24 \mathrm{~h}$ was enough for PAN double emulsions to completely become PAN spheres. Then the 24-hour period was equally divided into 6 blocks, namely, solidified capsules were collected after every 4 hours. Subsequently the corresponding dried PAN hollow capsules and hollow carbon spheres after carbonization were characterized using SEM, as shown in Fig. 4 and Fig. 5. 

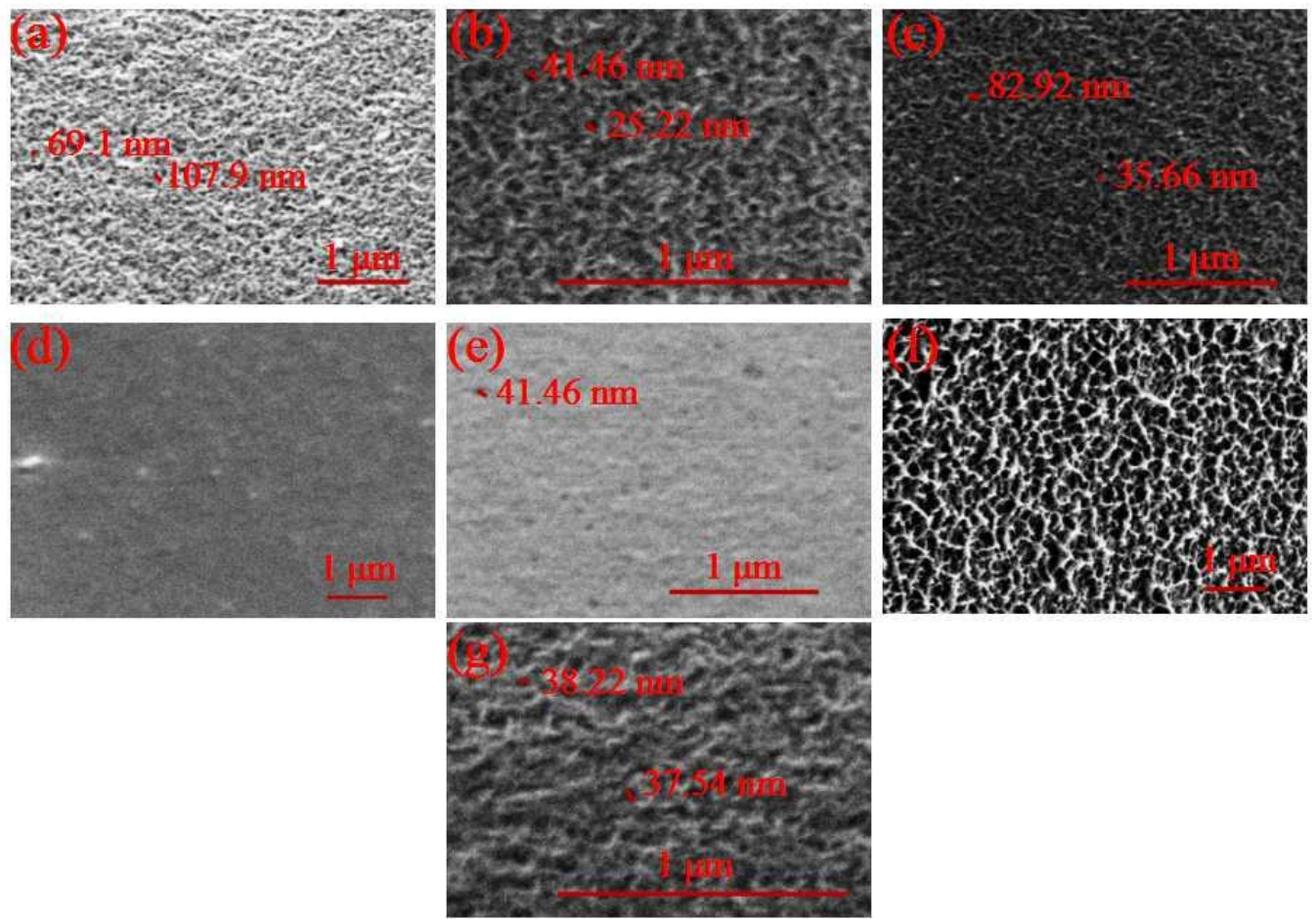

Fig.4. Cross-sectional SEM images of dried PAN hollow capsules synthesized by solidifying

for different time: (a): 0 h; (b): 4 h; (c): 8 h; (d): 12 h; (e): 16 h; (f): 20 h; (g): 24 h. Scale bars:

\section{$1 \mu \mathrm{m}$}
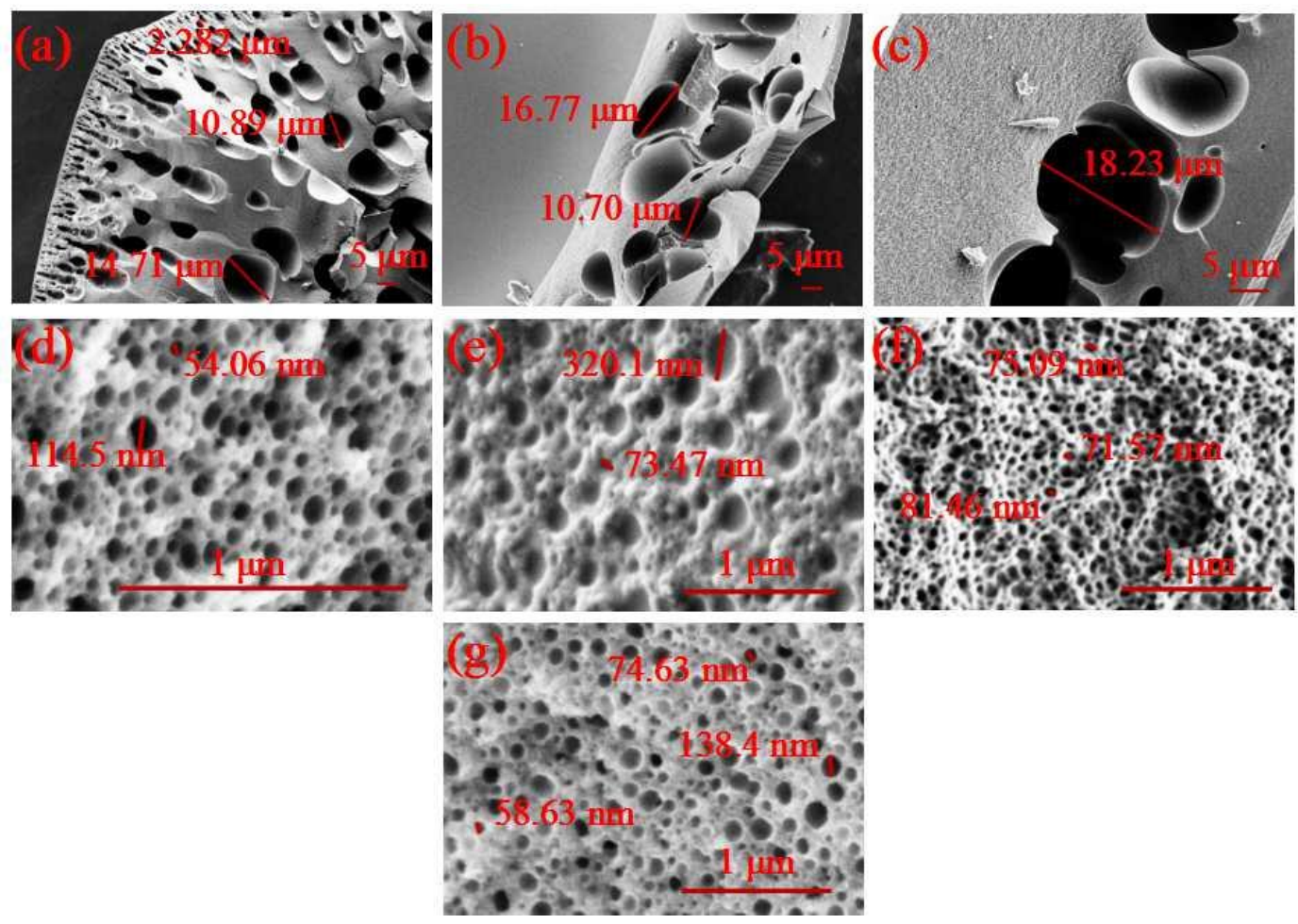

Fig.5. Cross-sectional SEM images of carbonized PAN hollow carbon spheres synthesized by 
solidifying for different time: (a): $0 \mathrm{~h}$; (b): $4 \mathrm{~h}$; (c): $8 \mathrm{~h}$; (d): $12 \mathrm{~h}$; (e): $16 \mathrm{~h}$; (f): $20 \mathrm{~h} ;(\mathrm{g}): 24 \mathrm{~h}$. (a)-(c) Scale bars: $5 \mu \mathrm{m}$ (d)-(g) Scale bars: $1 \mu \mathrm{m}$

One could find that white area in Fig. 4 was much larger than the one in Fig. 5. It was suggested that the electrical conductivity of hollow carbon spheres after carbonization was obviously higher than that of PAN hollow capsules. Because as a kind of polymer, PAN hollow capsules were similar to conventional plastic including physical and chemical properties such as the appearance and electrical conductivity [29]. On the other hand, based on the analysis of these SEM figures, we could easily find their pore structures and the holes of PAN capsules were much smaller and denser than those of the carbon spheres. It was reported that PAN pre-oxidized microspheres could form two kinds of pyrolysis components in the carbonization process: gas and another special phase component [30]. For one thing non-carbon elements were escaped in the form of gas and resulted in forming pores. For example, $\mathrm{N}$ was escaped in the form of $\mathrm{N}_{2}$ or $\mathrm{NH}_{3}$ or $\mathrm{HCN}, \mathrm{H}$ was escaped in the form of $\mathrm{H}_{2}$ or $\mathrm{H}_{2} \mathrm{O}$ vapor or $\mathrm{HCN}$, $\mathrm{O}$ was escaped in the form of $\mathrm{O}_{2}$ or $\mathrm{H}_{2} \mathrm{O}$ vapor. For another the above mentioned special phase component was carbonization tar produced in the process of carbonization. The special component was in the form of gas in high temperature and tar at room temperature. Carbonization tar was similar with the production of biomass pyrolysis and would affect the formation of pores.

It was also suggested in Fig. 4 and Fig. 5 that pore sizes of PAN hollow capsules and hollow carbon spheres decreased with increasing solidification time. When the solidification time was ranging from $0 \mathrm{~h}$ to $24 \mathrm{~h}$, the pore sizes of PAN hollow 
capsules were ranging from hundreds of nanometers to tens of nanometers and those of hollow carbon spheres were ranging from micrometers to nanometers. So it could be confirmed that the solidification time would obviously affect the density of PAN hollow capsules and hollow carbon spheres. However, with the solidification time became longer and longer, the pore sizes had not significant changes. And thus we reasonably assumed that great changes of pore sizes and porosities of PAN hollow capsules and hollow carbon spheres took place in the first 4 hours. In order to more easily find the regularity of solidification time and density of PAN hollow capsules, we shorten the solidification time to 4 hours. Similarly, the 4-hour period was equally divided into 8 blocks.

The morphology of PAN hollow capsules after rinsing with water for each time block was shown in Fig. 6. It was observed that there was a shallow ring inside the PAN capsules (Fig. 6e and 6h), which was likely to form a triple structure. This phenomenon was appeared because the internal solvent had not been completely removed. At this point, we reused hexane to wash the capsules for several more times, and then used acetone and water to repeat rinsing. A more recommended approach to avoiding shallow ring was to use gradient replacement before washing by hexane, namely, PAN hollow capsules were put into 10 cSt, 5 cSt, 1 cSt, 0.65 cSt KF-96 in turn for more than 8 hours after solidification in $350 \mathrm{cSt}$ KF-96. This approach of gradient replacement mainly had two advantages. First, it could avoid the formation of solid spheres because of emulsions hatching. PAN double emulsions could not form tough shells when solidified for a short time. At this point, silicone oil as the 
inner phase would be directly dissolved in hexane in the external environment, which resulted in forming solid capsules after drying. Second, it could guarantee to remove inner phase easily and completely in the following process. After gradient replacement, the PAN microcapsules were not easy to deform and could maintain better morphology. Less viscosity silicone oil in PAN capsules was easier to exchange with hexane, acetone and water. The morphology of obtained dried PAN hollow capsules and hollow carbon spheres was shown in Supplementary Fig.S2 and S3, respectively. The structure of the hollow carbon spheres exhibited a dense skin layer, a porous layer, and a less porous layer (Fig. 7). There were much more small pores across gaps between big pores on the side wall and no pores across gaps between small pores on the inner wall of hollow carbon spheres.
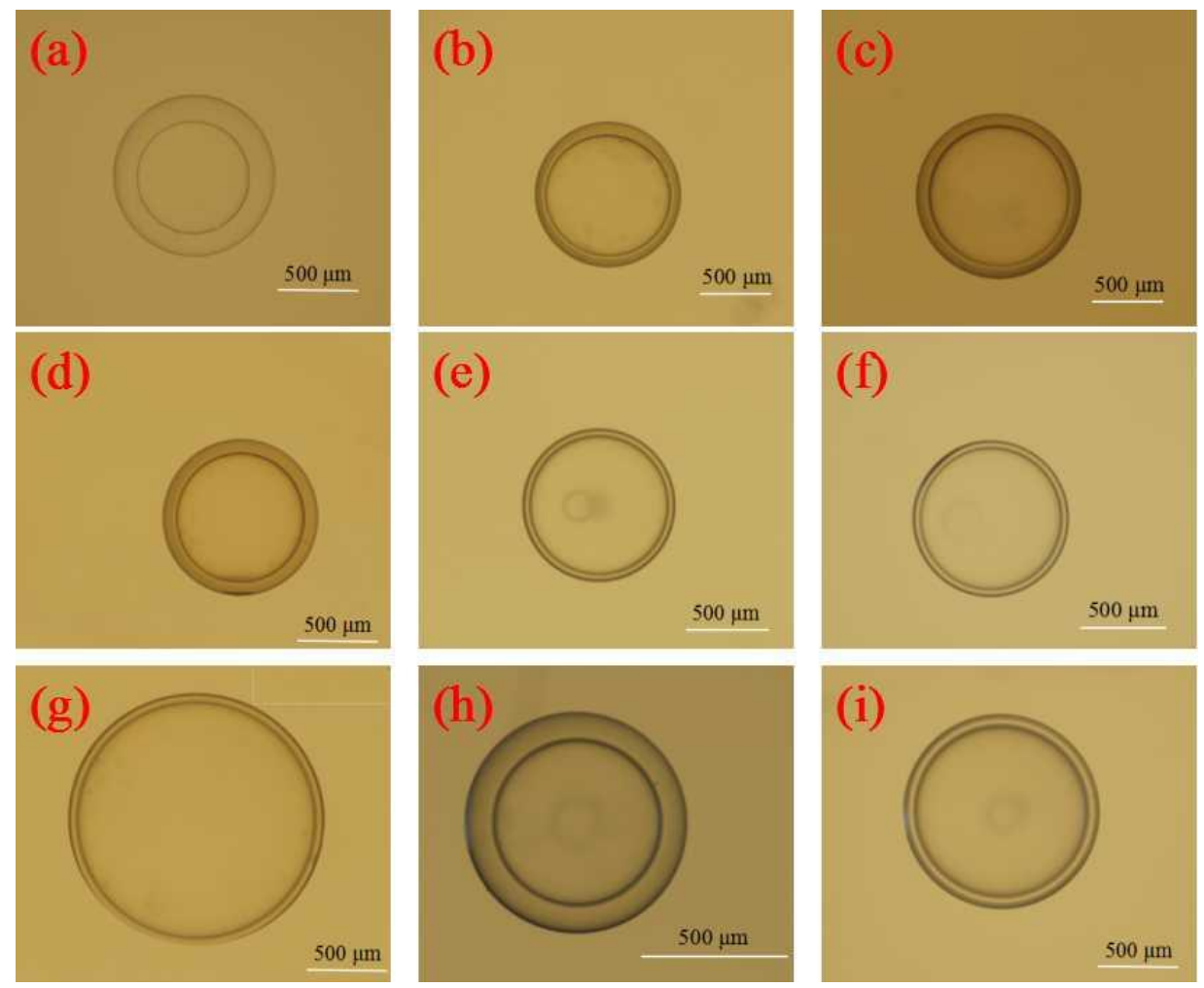

Fig.6. Optical images of PAN hollow microspheres immersed in water, which were synthesized by solidifying for different time: (a): $0 \mathrm{~h}$; (b): $0.5 \mathrm{~h}$; (c): $1 \mathrm{~h}$; (d): $1.5 \mathrm{~h} ;(\mathrm{e}): 2 \mathrm{~h} ;(\mathrm{f}): 2.5 \mathrm{~h} ;(\mathrm{g}): 3 \mathrm{~h}$; (h): 

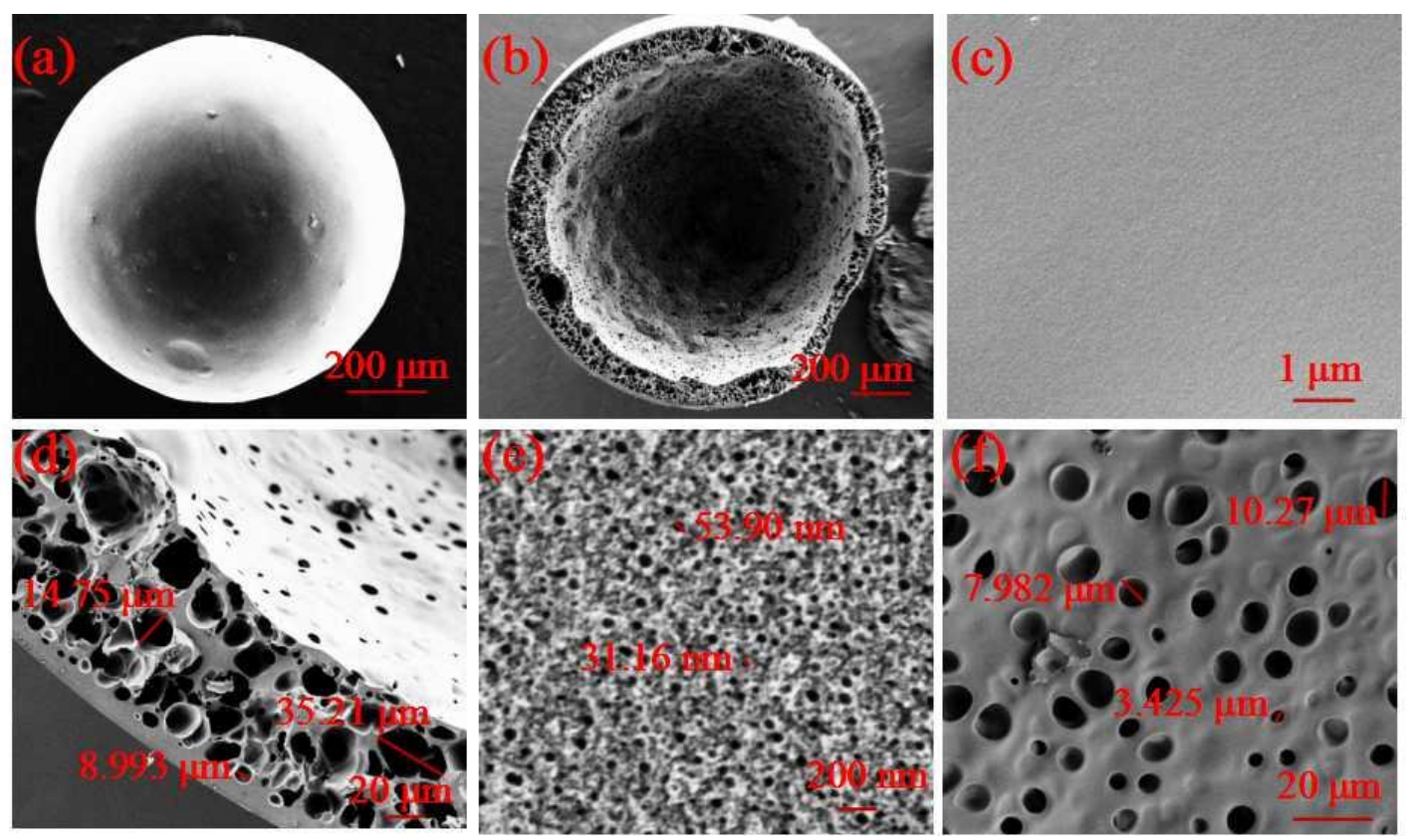

Fig.7. SEM images of obtained hollow carbon microspheres $\left(\mathrm{HCM}_{\mathrm{S}}\right)$. (a) The whole $\mathrm{HCM}_{\mathrm{S}}$.

Solidification time (ST): $8 \mathrm{~h}$. (b) A half of $\mathrm{HCM}_{\mathrm{S}}$ after cut. ST: $3.5 \mathrm{~h}$ (c) The outer wall of $\mathrm{HCM}_{\mathrm{S}}$.

ST: 0h. (d) The cross-sectional wall of $\mathrm{HCM}_{\mathrm{S}}$. ST: $3.5 \mathrm{~h}$ (e) The cross-sectional part located

between big pores of $\mathrm{HCM}_{\mathrm{S}}$. ST: 3.5h (f) The inner wall of HCM. ST: 3.5h (a)(b) Scale bars: 200

$\mu \mathrm{m}$; (c) Scale bar: $1 \mu \mathrm{m}$; (d)(f) Scale bars: $20 \mu \mathrm{m}$; (e) Scale bar: $200 \mathrm{~nm}$

Moreover, we found other interesting phenomenons, which were pointed out separately in Fig. 8. Some researches indicated that mass transfer through the capsule shell was strongly dependent on the shape, orientation, and uniformity of the pores [31]. So the excellent properties of pores were critical. Fortunately, our obtained hollow carbon microspheres were shown fantastic tunable pore structures -- gradient pore diameters and uniform orientation of pores. First, the pore sizes of hollow carbon spheres obeyed the law of radial distributions (Fig. 8a). Hollow carbon microspheres showed a gradient in their pore size with the smaller pores in the denser outer part and 
larger pores in the inner part. To put it another way, the finger-like holes became larger and deeper towards the center of spheres, which resulted in forming the sponge-like holes. The closer it got to outer wall, the more completely interaction with water and faster rate of removing DMF would be achieved. And during the process of pre-oxidation and carbonization, the position closer to outer wall suffered more sufficient heat, which resulted in more gas escaping from wall of carbon spheres. As a result, the porosity of carbon spheres towards outer wall was improved, and the pore size was smaller. Second, the pores of hollow carbon spheres had a uniform orientation (Fig.8b).The reasons for pore orientations were usually solvent effect, surfactant effect and porogens effect. In this paper, the solvent effect was the only reason for pore orientations. When rinsing with water, DMF and water were exchanged with each other resulting in forming pores. And the direction of solvents-exchange was further expanded to form pore orientation after carbonization. Finally, PAN hollow capsules after drying were likely to form a dense cross-linked network structure (Fig. 8c and 8d). The pores of PAN hollow capsules were so shallow and small that we considered that they were not yet formed completely. In other words, the dried PAN hollow capsules showed a tendency of forming pores. After pre-oxidation and carbonization, pores would further grow up and enlarge along the axial direction of the network, forming well-developed three-dimensionally interconnected pores. 

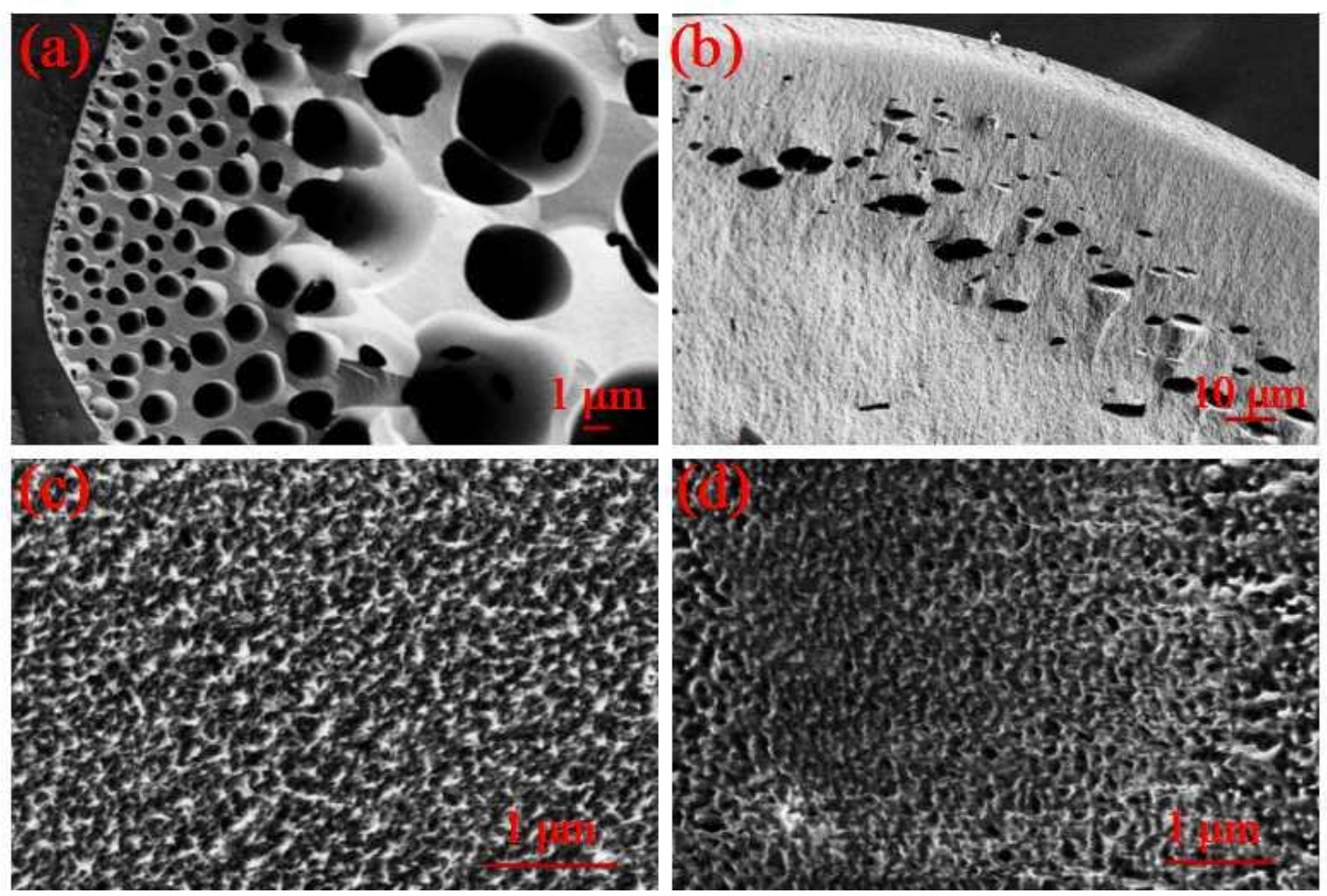

Fig.8. Cross-sectional SEM images of (a)(b): obtained hollow carbon microspheres and (c)(d):

dried PAN hollow capsules. (a) Solidification time (ST): $2 \mathrm{~h}$ (b) ST: 20h. (c) ST: 2h. (d) ST: 3.5h.

(a)(c)(d) Scale bars: $1 \mu \mathrm{m}$; (b) Scale bar: $10 \mu \mathrm{m}$.

\subsection{Density of the PAN microspheres}

The porous structures of PAN hollow capsules and hollow carbon spheres were achieved successfully according to the results of SEM, which were intuitive picture descriptions. In addition, in order to improve the rigor and objectivity of our experiment, we also needed digital description such as the density of PAN hollow capsules. So a number of samples were taken to obtain the average density of these hollow carbon capsules with different solidification time. The outer radius and inner radius of PAN hollow capsules could be easily measured by optical microscope, but those of hollow carbon microspheres could not be accurately measured due to the limitations of the experimental instrument and test cost. Therefore herein we only 
explored the density of PAN hollow capsules, where the trend of results should be theoretically consistent with that of hollow carbon microspheres. From Fig. 9, it was shown that the density of PAN hollow capsules increased with the solidification time. The density was almost lowest for PAN hollow capsules prepared by rinsing with water after solidification less than 1 hour (Supplementary Table S2). So we could draw a conclusion that the solidification time had a major influence on the density of PAN hollow capsules. A same relationship between the solidification time and density of hollow carbon microcapsules was deducted. And we hypothesized that the density of hollow carbon microcapsules was lower than that of PAN hollow capsules because more materials transformed into gas after pre-oxidation and carbonization.

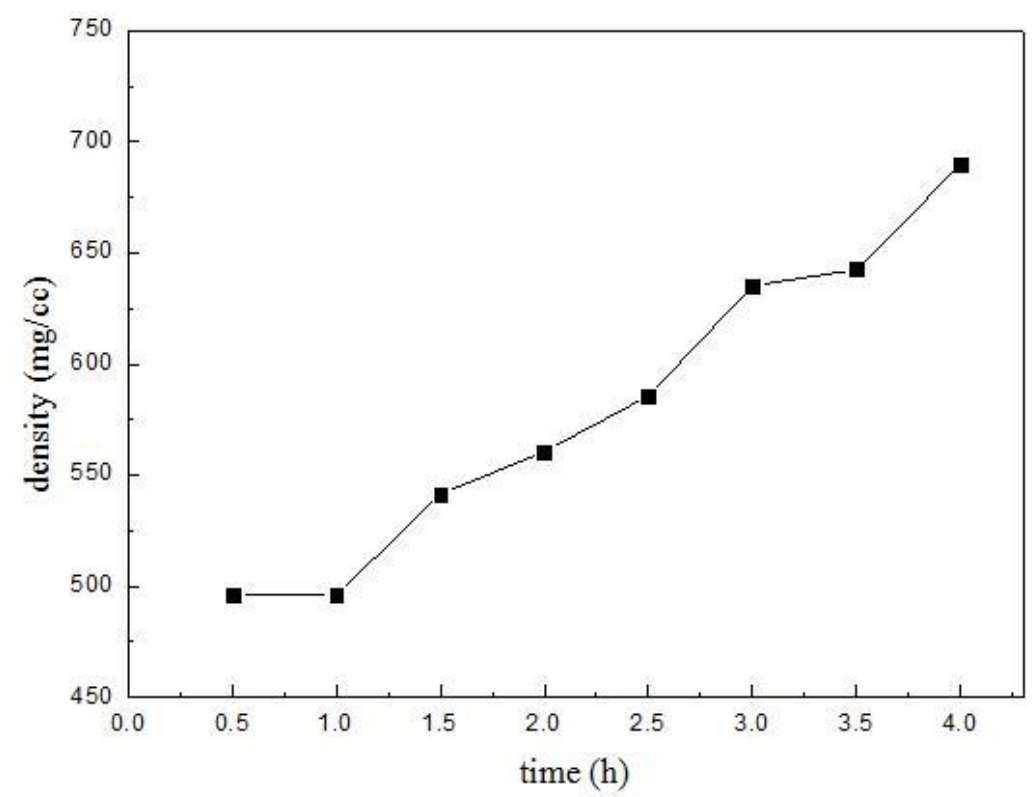

Fig.9. Effects of solidification time on density of PAN hollow microspheres.

\subsection{Simulation of gas permeation rate of PAN capsules}

Due to the limitations of laboratory equipment, gas permeation rate of PAN microcapsules and corresponding carbon spheres could not be directly measured. So we measured the gas transmission rate of PAN membranes to simulate that of PAN 
microspheres with the help of Institute of Membrane Science and Technology, Nanjing Tech University. At first we used the same $0.1 \mathrm{~g} / \mathrm{ml}$ PAN solution to prepare PAN self-support membranes. However, it was known that PAN featured super water-absorbing. PAN solutions became gels when exposed to air for 10-30 min, so the viscosity of $0.1 \mathrm{~g} / \mathrm{ml}$ PAN solution was too high to obtain PAN membranes. Then we reduced the concentration of PAN solution to $0.05 \mathrm{~g} / \mathrm{ml}$.

Different gases permeability coefficient of PAN thin membranes were shown in Fig. 10. It was clearly seen that hydrogen permeability coefficient was the lowest (Supplementary Table S3). And the permeability coefficient of nitrogen, the main components of air, was lower. It suggested that PAN membranes had perfect gas barrier properties. The reduction of gases permeability was attributed to the formation of a cross-linked network produced under dry conditions [32], which was similar to PAN dried capsules as illustrated in Fig. 8c and 8d.

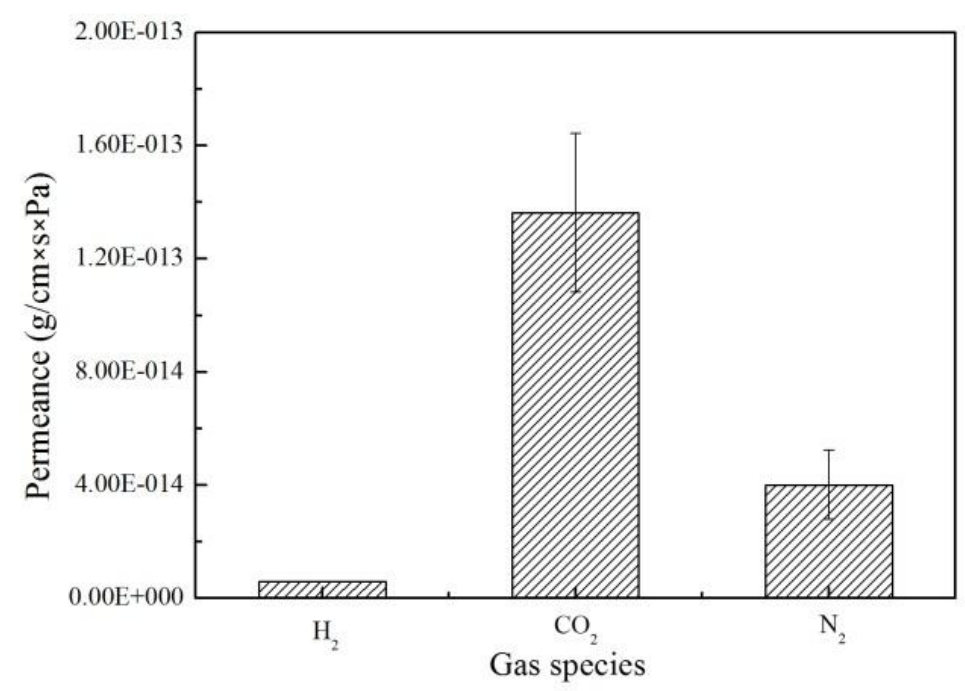

Fig.10. Results of the gas permeation tests

The permeability coefficient was used as the evaluation index of gas permeability, 
as shown in equations (3), (4). Combing equations (3) with (4), equation (5) could be obtained, from which we could see that the selective gas permeability coefficient was mainly determined by the characteristics of tested materials and molar mass of tested gas for the same sample under the same experimental environment.

$$
\mathrm{P}_{\mathrm{v}}=\frac{\mathrm{m} * \mathrm{~d}}{\mathrm{~A} * \mathrm{t} * \mathrm{p}}=\frac{\mathrm{V}}{\mathrm{V}_{\mathrm{m}} * \mathrm{~A} * \mathrm{P}} * \frac{\mathrm{d}}{\mathrm{t}} * \mathrm{M}
$$

Where $P_{V}, V, V_{m}, m, d, A, t, p, M$ are the permeability coefficient, the volume of gas permeated, molar volume of gas, the mass of gas permeated, the thickness of the membrane, the area of gas pass through the membrane, the time of gas permeated, pressure difference on both sides of the membrane, molar mass respectively.

On the one hand, the characteristics of tested materials determined the velocity of different gas molecules permeated, namely, $d / t$ the time of gas permeated through same distance. The characteristics of tested materials included the affinity and interaction between tested gas and materials, the molecular structure of polymer materials (the primary structure) and the preparation conditions (the advanced structure) such as preparation temperature and crystallinity. For example, the permeability coefficient of $\mathrm{H}_{2}$ for tested PAN-based material was the lowest because PAN had low permeability of hydrogen and hydrogen isotopes, which was confirmed in the previous research of target materials [16]. On the other hand, the molar mass of tested gas determined the mass of gas permeated under the same experimental environment. The larger molar mass of tested gas led the increased gap of the gas permeability coefficient. For example, the difference in the velocity of $\mathrm{CO}_{2}$ and $\mathrm{H}_{2}$, $\mathrm{N}_{2}$ was further expanded by multiplying molar mass, which led the increased gap of 
the gas permeability coefficient between $\mathrm{CO}_{2}$ and $\mathrm{H}_{2}, \mathrm{~N}_{2}$.

In addition we could deduce that the PAN capsules and corresponding carbon spheres also had perfect gas barrier properties as PAN membranes. Except the formation of the network structure during the drying process, there were other three factors. First, the chemical structure of PAN had been changed during the process of pre-oxidation and carbonization. PAN was changed from linear polymer into a trapezoidal structure with conjugate rings by cyclization, dehydrogenation and oxidation reaction in pre-oxidation (Fig. 11). And then when exposed to high temperature in carbonization, PAN was formed into cross-linked carbon network structure by intermolecular dehydration and dehydrogenation. As the carbonization process unfolded, the carbon network was further expanded [33]. The large network could prevent gas from diffusing through shells of carbon microspheres. Second, the skin-core structure would be formed during the process of pre-oxidation [29, 34]. Along with different extents of reactions, different skin-core structures were generated. But these structures had something in common. It was that the cortex represented fully stable polymers while the core was composed of unstable or partially stable polymers. The formation of cortex would hinder gas inside PAN microspheres diffusing to the external environment. Third, the dense outer wall and carbonization tar were formed during the process of carbonization. The dense outer wall and porous structures lessened the area available for the diffusion of gases and meanwhile led to a tortuous pathway for gas with a consequent improvement of barrier properties [32] (Fig. 7). Previous works had reported that the carbonization tar was centrally formed 
in the range of $450-780{ }^{\circ} \mathrm{C}$ and the main element of carbonization tar was carbon [30]. There were nitrogen and oxygen elements on the surface of PAN carbon spheres, but there was only carbon element in carbonization tar. Therefore, the existence of carbonization tar could reduce the spread rate of gas in theory.

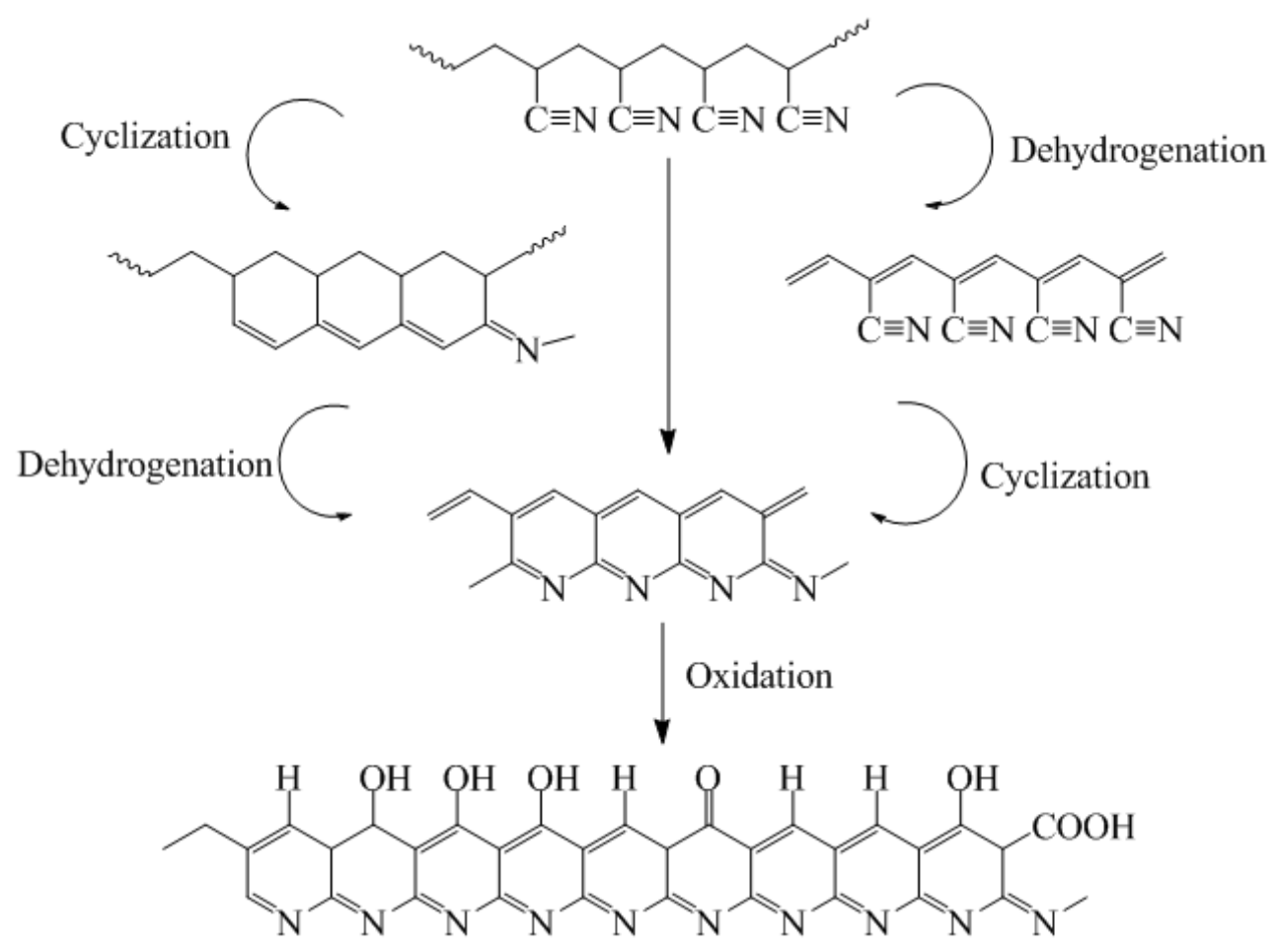

Fig.11. Scheme of pre-oxidation reaction.

\section{Conclusions}

In summary, we have fabricated porous hollow PAN microcapsules and corresponding carbon spheres with high monodispersity, defined size and orientation of pores by tuning the solidification time and then directly rinsing with water to remove DMF solvent. The flowing rates of three phases when preparing double emulsions were tunable in terms of the desirable size of microspheres. The novel method has been proved to be a simple, easy, low-cost way which can be utilized for 
the mass production. Next, our goal is to combine solidification time with porogens to control the porous structure. Furthermore, we have considered that this material would be used as potential porous foam targets $[16,35]$ on account of its hydrogen barrier properties with low $\mathrm{H}_{2}$ permeability coefficient. In addition, the methods and products we described herein have potential applications in other various areas, including selective filtration, carbon molecular sieve, microfluidic reactors, cargo storage, medical instruments, drug carriers, aerospace materials and bio-chemistry.

\section{Acknowledgements}

The authors thank for the financial support of Pre-research Foundation for National Science Foundation and the Science and the support from Prof. Dr. Z.Z. Gu's group.

\section{References}

[1] L. Cheng, Y. Liu, J. Zhang, D. Yuan, C. Xu, G. Sun, Synthesis and application of spherical structured carbon materials, Progress in Chemistry 10 (2006) 1298-1304.

[2] G. Yang, R. Xu, M. Chen, X. Wang, L. Ling, R. Zhang, J. Yang, Hollow carbon nanospheres prepared by carbonizing polymethylmethacrylate/polyacrylonitrile core/shell polymer particles, New Carbon Materials 23 (2008) 205-208.

[3] J. Tang, J. Liu, R. R. Salunkhe, T. Wang, Y. Yamauchi, Nitrogen-doped HCS with large mesoporous shells engineered from diblock copolymer micelles, Chem. Commun. 52 (2016) 505-508.

[4] Q. Ma, L. Wang, W. Xia, D. Jia, Z. Zhao, Nitrogen-doped hollow amorphous carbonspheres@graphitic shells derived from pitch: new structure leads to robust 
lithium storage, Chem. Eur. J. 22 (2016) 1-7.

[5] C. J. Hofer, R. N. Grass, M. Zeltner, C. A. Mora, F. Krumeich, W. J. Stark, Hollow carbon nanobubbles: synthesis, chemical Functionalization, and container-type behavior in water, Angew. Chem. Int. Ed. 55 (2016) 1-6.

[6] M. Dai, Y. Wang, M. Pan, L. Garry, Rempel, Q. Pan, Progess on preparation of hollow/porous microspheres, Materials Review 27 (2013) 80-86.

[7] Y. Wang, Q. Zhang, M. Ma, C. Yin, X. Kou, New Advances in pore-forming mechanisms of porous polymer particles, Materials Review 25 (2011) 88-92.

[8] C. Liu, Y. Yun, N. Wu, Y. Hua, C. Li, Effects of amphiphilic additive Pluronic F127 on performance of poly (ether sulfone) ultrafiltration membrane, Desalination and Water Treatment 51 (2013) 3776-3785.

[9] J. Yao, H. Wang, J. Liu, K. Chan, L. Zhang, N. Xu, Preparation of colloidal microporous carbon spheres from furfuryl alcohol, Carbon 43 (2005) 1709-1715.

[10] H. Xu, J. Guo, K. S. Suslick, Porous Carbon Spheres from Energetic Carbon Precursors using Ultrasonic Spray Pyrolysis, Adv. Mater. 24 (2012) 6028-6033.

[11] K. Zhang, X. Feng, X. Sui, M. A. Hempenius, G. J. Vancso, Breathing pores on command: redox-responsive spongy membranes from poly(ferrocenylsilane)s, Angew. Chem. Int. Ed. 53 (2014) 1-6.

[12] J. Shi, H. Wang, X. Zhu, C. Chen, X. Huang, X. Zhang, B. Li, C. Tang, Q. Zhang, The nanostructure preservation of 3D porous graphene: new insights into the graphitization and surface chemistry of non-stacked double layer templated graphene after high-temperature treatment, Carbon 103 (2016) 36-44. 
[13] Z. Li, X. Ma, Q. Hong, X. Guan, Functional applications of ordered honeycomb-patterned porous films based on the breath figure technique, Acta Phys. -Chim. Sin. 31 (2015) 393-411.

[14] K. Täuber, B. Lepenies, J. Yuan, Polyvinylpyridinium-type gradient porous membranes: synthesis, actuation and intrinsic cell growth inhibition, Polym. Chem. 6 (2015) 4855-4858.

[15] H. Peng, Z. Xu, S. Chen, Z. Zhang, B. Li, L. Ge, An easily assembled double T-shape microfluidic devices for the preparation of submillimeter-sized polyacronitrile (PAN) microbubbles and polystyrene (PS) double emulsions, Colloids and Surfaces A: Physicochem. Eng. Aspects 468(2015) 271-279.

[16] H. Peng, Z. Shi, W. Wang, S. Chen, Z. Zhang, Z. Xu, S. Dong, Y. Chen, B. Li, L. Ge, An easily assembled double T-shape for the preparation of submillimeter-sized hollow polyacrylonitrile (PAN) microcapsules for inertial confinement fusion (ICF) project, Colloids and Surfaces A: Physicochem. Eng. Aspects 482 (2015) 58-67.

[17] D. S. Mahrhauser, C. Fischer, C. Valenta, Double emulsions based on silicone-fluorocarbon-water and their skin penetration, International Journal of Pharmaceutics 498 (2016) 130-133.

[18] Y. Wang, T. Zhang, G. Hu, Structural evolution of polymer-stabilized double emulsions, Langmuir 22 (2006) 67-73.

[19] D. Liu, B. Hakimi, M. Volny, J. Rolfs, X. Chen, F. Turecek, D. T. Chiu, Controlled generation of double emulsions in air, Anal. Chem. 85 (2013) 6190-6194.

[20] M. C. Paez, C. M. Quezada, L. I. Bracamontes, H. O. G. Ochoa, J. A. Lara, 
Coalescence in double emulsions, Langmuir 28 (2012) 5934-5939.

[21] X. Gong, Y. Lu, Z. Xiang, G. Luo, Preparation of microcapsules with silicone oil as continuous phase using a solvent evaporation method, Acta Polymerica Sinica 7 (2007) 775-779.

[22] H. H. Jeong, V. R. Yelleswarapu, S. Yadavali, D. Issadore, D. Lee, Kilo-scale Droplet generation in three-dimensional monolithic elastomer device, Lab Chip 15 (2015) 4387-4392.

[23] B. U. Moon, S. G. Jones, D. K. Hwang, S. S. H. Tsai, Microfluidic generation of aqueous two-phase system (ATPS) droplets by controlled pulsating inlet pressures, Lab Chip 15 (2015) 2437-2444.

[24] H. Breisig, M. Wessling, Droplet formation and shrinking in aqueous two-phase systems using a membrane emulsification method, Biomicrofludics 9 (2015) 143-152. [25] P. Wu, Y. Wang, Z. Luo, Y. Li, M. Li, L. He, A 3D easily-assembled micro-cross for droplet generation, Lab on a chip 14 (2013) 795-798.

[26] N. P. B. Tan, L. H. Keung, W. H. Choi, W. C. Lam, H. N. Leung, Silica-based self-healing microcapsules for self-repair in concrete, J. Appl. Polym. Sci. 133 (2016) n/a-n/a.

[27] J. Zhang, R. J. Coulston, S. T. Jones, J. Geng, O. A. Scherman, C. Abell, One-step fabrication of supramolecular microcapsules from microfluidic droplets, Science 335 (2012) 690-4.

[28] M. Kim, S. J. Yeo, C. B. Highley, J. A. Burdick, P. J. Yoo, J. Doh, D. Lee, Nanoscale interfacial complexation in emulsion (NICE), ACSNANO 8 (2015) 
8269-8278.

[29] X. Cai, The production technology of polyacrylonitrile-based carbon fiber, first ed., Chemical Industry Press, Beijing, 2012.

[30] Q. Wang, B. Zhu, X. Gao, C. Wang, Reasons for tar formation from polyacrylonitrile-based carbon fiber, China Synthetic Fiber Industry 3 (2005) 37-39.

[31] J. Xu, J. Li, Y. Yang, K. Wang, N. Xu, J. Li, R. Liang, L. Shen, X. Xie, J. Tao, J. Zhu, Block copolymer capsules with structure-dependent release behavior, Angew. Chem. Int. Ed. 55 (2016) 1-6.

[32] N. Yan, F. Capezzuto, M. Lavorgna, G. G. Buonocore, F. Tescione, H. Xia, L. Ambrosioa, Borate cross-linked graphene oxide-chitosan as robust and high gas barrier films, Nanoscale 8 (2016) 10783-10791.

[33] J. Liu, N. P. Wickramaratne, S. Qiao, M. Jaroniec, Molecular-based design and emerging applications of nanoporous carbon spheres, Nature Materials 14 (2015) 763-774.

[34] E. A. Morris, M. C. Weisenberger, M. G. Abdallah, F.Vautard, H. Grappe, S. Ozcan, F. L. Paulauskas, C. Eberle, D. Jackson, S. J. Mecham, A. K. Naskar, High performance carbon fibers from very high molecular weight polyacrylonitrile precursors, Carbon 101 (2016) 245-252.

[35] J. Tang, Z. Xie, A. Du, J. Ye, Z. Zhang, J.Shen, B. Zhou, Design and fabrication of a $\mathrm{CH} / \mathrm{RF} / \mathrm{CH}$ tri-Layer perturbation target for hydrodynamic instability experiments in ICF, J Fusion Energ 35 (2016) 357-364. 


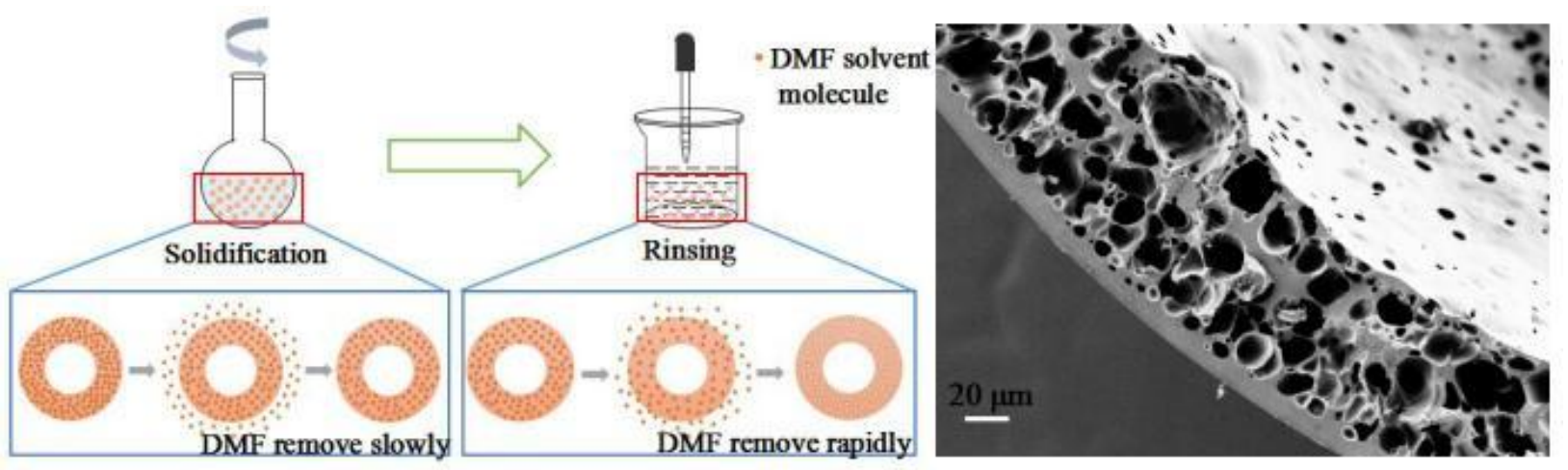

